\title{
Medicamentos Potencialmente Perigosos: Como Garantir a sua Segurança no Hospital
}

\section{High Alert Medications: How to Ensure its Safety in the Hospital}

Leonor Mira', Sílvia Martins ${ }^{1}$

Autor Correspondente:

\section{RESUMO}

Os medicamentos de alerta máximo são caracterizados por um risco acrescido de provocar dano significativo ao doente em consequência de falhas no seu processo de utilização. Neste sentido, este artigo de revisão procura definir procedimentos para reduzir o risco de erros associados à utilização de medicamentos look-alike, sound-alike (LASA) e concentrados eletrolíticos. Os profissionais de saúde, doentes e seus cuidadores devem ser formados e elucidados sobre os medicamentos de alerta máximo.

PALAVRAS-CHAVE: Erros de Medicação/prevenção e controlo; Hospitais; Medicamentos sob Prescrição; Rotulagem de Medicamentos; Segurança do Doente

\section{ABSTRACT}

High-alert medications are characterized by an increased risk of causing significant harm to the patient as a result of failures in their use process. This review finds to establish procedures to reduce the risk of errors associated with the use of look-alike, sound-alike (LASA) and electrolytic concentrates. Healthcare professionals, patients and their caregivers should be educated and elucidated about the high-alert medications.

KEYWORDS: Drug Labeling; Hospitals; Medication Errors/prevention \& control; Patient Safety; Prescription Drugs 


\section{INTRODUÇÃO}

Os erros de medicação são um importante e grave problema de saúde pública que pode originar consequências quer a nível humano, quer económico. Um erro de medicação tem por definição, um evento prevenível, que pode resultar do uso inadequado de medicamentos ou causar danos ao doente colocando em causa a sua segurança. Este erro de medicação pode ocorrer ao longo de todo o circuito do medicamento, ou seja, na prescrição, transcrição, interpretação, dispensa ou administração. ${ }^{1,2}$

O conceito de medicamentos de alto risco ou alerta máximo (AM) nasceu nos Estados Unidos da América em 1998 pelo Institute for Safe Medication Practices (ISMP), após a realização de um estudo em 161 hospitais, em que se verificou que um número reduzido de medicamentos causava a maioria dos erros de medicação com consequências devastadoras para os doentes. A informação recolhida no estudo e os casos notificados levaram o ISMP a definir uma lista de medicamentos classificados como medicamentos de alto risco em meio hospitalar. Esta lista tem vindo a ser atualizada periodicamente. ${ }^{3}$

Os medicamentos de AM apresentam um risco acrescido, ou maior probabilidade, de causar danos graves ao doente quando utilizados de forma incorreta, mas não significa que os erros com estes medicamentos sejam mais frequentes, contudo se estes ocorrerem as consequências tendem a ser mais graves para os doentes, levando muitas vezes a lesões permanentes ou mesmo à morte., ${ }^{4,5}$

Os medicamentos de AM incluem os medicamentos look-alike, medicamentos sound-alike e concentrados eletrolíticos. Os medicamentos com nome ortográfico e/ou fonético e/ou aspeto semelhante, designam-se por medicamentos LASA (look-alike, sound-alike), que podem ser confundidos uns com os outros, originando troca entre os mesmos: medicamentos com aspeto ou ortografia semelhante são chamados de medicamentos look-alike; medicamentos com nome foneticamente semelhante, são chamados medicamentos sound-alike. Por sua vez, os concentrados eletrolíticos caracterizam-se por apresentar elevada concentração em iões livres, sendo utilizados na prática clínica com o objetivo de corrigir défices no equilíbrio hidroeletrolítico do organismo. ${ }^{1,6}$

A Direção-Geral de Saúde (DGS) desenvolveu uma lista de medicamentos LASA (medicamentos com ortografia semelhante ou fonética semelhante), tendo em conta a Denominação Comum Internacional (DCI), e com base nos seguintes documentos internacionais, "ISMP's List of Confused Drug Names" (ISMP 2010), "Nuevos pares de nombres de medicamentos que se prestan a confu- sión por similitude ortográfica y/o fonética" (ISMP España 2010), "Lista de nomes de medicamentos com grafia ou som semelhantes" (ISMP Brasil 2014), e "National Standard for the Application of Tall Man Lettering: Project Report" (Australian Comission on Safety and Quality in Health Care 2011).

O método Tall Man Lettering é um método baseado na inserção de letras maiúsculas no meio das denominações dos medicamentos com ortografia semelhante, como exemplo temos o alopurinol e haloperidol que aplicando o método Tall Man Lettering fica aloPURINol e halOPERIDol. ${ }^{6}$

O facto de existirem medicamentos com nomes semeIhantes faz com que esta seja uma das maiores causas de erros de medicação. Assim, a indústria farmacêutica tem também desenvolvido medidas de forma a evitar a existência de semelhanças inaceitáveis nos nomes dos medicamentos. ${ }^{2,7}$

\section{RECOMENDAÇÕES PARA A PREVENÇÃO DE ERROS ASSOCIADOS AOS MEDICAMENTOS DE ALERTA MÁXIMO}

O ISMP e outras organizações focadas na segurança do doente têm vindo a desenvolver estratégias para minimizar o risco de ocorrência de erros relacionados com este grupo de medicamentos. É recomendável:

- Promover uma cultura de segurança, sensibilizando as equipas para possíveis erros que possam vir a acontecer. Se o erro se verificar, implementar ações corretivas e definir procedimentos que dificultem ou que tornem impossíveis a repetição do erro;

- Elaborar protocolos institucionais de administração de medicamentos;

- Identificar, listar e divulgar os medicamentos de AM existentes no hospital e definir circuitos para a sua utilização segura;

- Reduzir o número de apresentações, quer em quantidade, quer em variedade, e de concentrações de medicamentos de AM, aquando da aquisição dos medicamentos;

- Aumentar o acesso à informação destes medicamentos, tanto para profissionais de saúde, como para os doentes e seus cuidadores;

- Usar etiquetas sinalizadoras para destacar os medicamentos de AM dos restantes e, se possível, limitar o seu acesso no stock dos serviços; 
- Recorrer sempre à prescrição eletrónica e proibir o uso de abreviaturas nas prescrições manuais. Disponibilizar informação e alertas, como exemplo, limites de dose ou necessidade de diluição;

- Proceder à validação da prescrição médica e posterior distribuição em dose unitária;

- Estabelecer doses máximas e parametrizar alertas para os medicamentos de AM (como exemplo, interações com outros medicamentos, alergias);

- Centralizar a preparação de misturas intravenosas nos serviços farmacêuticos, processos com elevado potencial de indução de erro (como exemplo a preparação de citotóxicos);

- Garantir o cumprimento dos 9 certos de preparação e administração de medicamentos. Reforçar a dupla verificação na preparação e administração de medicamentos de AM, LASA e concentrados eletrolíticos;

- Informar os doentes e/ou cuidador principal sobre este grupo de medicamentos e possíveis erros que possam ocorrer.1, ${ }^{1,5}$

\section{CLASSES DE MEDICAMENTOS DE ALERTA MÁXIMO}

As classes de medicamentos e exemplos enunciados abaixo, constituem a lista dos medicamentos potencialmente perigosos devido à sua probabilidade de eventos adversos causados por erros de administração. Nesta lista incluem-se também algumas estratégias de prevenção e precauções associadas a este grupo de medicamentos:

- Adrenérgicos intravenosos, como exemplos: adrenalina, dobutamina, dopamina, isoprenalina, noradrenalina;

- Anestésicos gerais administrados por via inalatória e intravenosa: cetamina, desflurano, etomidato, isoflurano, propofol, sevoflurano;

- Antagonistas adrenérgicos, esmolol, propranolol;

- Antiarrítmicos intravenosos: adenosina, amiodarona, atropina, esmolol, flecainida, lidocaína, propafenona, vernacalant;

- Inotrópicos intravenosos: amrinona, digoxina, inosina, levocarnitina, levossimendano, milrinona;

- Meios de contraste intravenoso;

- Medicamentos intravenosos para indução de sedação moderada; dexmedetomidina, midazolam;

- Insulinas intravenosas e subcutâneas e antidiabéticos orais: formação dos profissionais de saúde para o uso correto destas classes de medicamentos. Aquando da prescrição de insulinas escrever "unidades", em vez de apenas "U";

- Bloqueadores neuromusculares, suxametónio, rocurónio, vecurónio. Limitar este grupo de medicamentos apenas aos serviços de urgência e blocos operatórios;

- Citotóxicos intravenosos ou orais, todos os medicamentos citotóxicos são considerados medicamentos de alerta máximo. Deve existir sempre dupla verificação destes procedimentos farmacêuticos e, recorrer-se ao uso de embalagens e rótulos especiais para esta classe de medicamentos;

- Medicamentos para administração por via epidural ou intratecal, uma das medidas passa pela colocação de etiquetas que indiquem "uso exclusivo intratecal" ou "uso exclusivo epidural";

- Opioides intravenosos, orais e transdérmicos, ter disponível, caso se justifique o uso de naloxona nos serviços onde são utilizados opioides. Existir formação sobre o uso dos adesivos de fentanilo e de protocolos de tratamento que incluam as doses máximas dos opioides;

- Soluções concentradas de eletrólitos, restringir o acesso das soluções de concentrados eletrolíticos nas enfermarias. Uso de protocolo para a administração dos concentrados eletrolíticos;

\section{- Soluções para hemodiálise e diálise peritoneal;}

- Soluções cardioplégicas;

- Agentes antitrombóticos, anticoagulantes, como, a heparina de baixo peso molecular (enoxaparina), a heparina não fracionada ou a varfarina. Ainda fazem parte desta lista os antiagregantes plaquetários, os inibidores do fator $\mathrm{X}$, os inibidores diretos da trombina e os trombolíticos, como o alteplase, reteplase e tenecteplase. Na heparina e à semelhança das insulinas, escrever sempre "unidades" em vez "U" e desenvolver protocolos de monitorização do índice internacional normalizado (INR). ${ }^{1,5,8,9}$

Assim, e com base em 317 incidentes evitáveis relacionados com a medicação, constatou-se que mais de 50\% dos casos ocorriam devido a: sobredosagem de anticoagulantes ou insuficiente monitorização e ajustes posológicos associados a eventos hemorrágicos; sobredosagem ou falha em identificar interações com agonistas opiáceos, associados a sonolência e depressão respiratória, e doses inapropriadas ou monitorização insuficiente de insulinas, associadas a hipoglicemia. ${ }^{10}$

Por sua vez, a tabela apresentada em seguida, tabela de medicamentos específicos (Tabela 1) contempla apenas 
TABELA 1. Medicamentos específicos.

Água estéril para inalação e irrigação (embalagens de volume igual
ou superior a $100 \mathrm{~mL}$ )
Cloreto de sódio hipertónico solução injetável (superior a 0,9\%)
Cloreto de potássio concentrado de solução para perfusão
Glicose hipertónica injetável (superior ou igual a 20\%)
Fosfato monopotássico solução injetável
Sulfato de magnésio solução injetável
Metotrexato comprimido
Anfotericina B (convencional ou lipossómica)
Epoprostenol
Oxitocina solução injetável
Prometazina solução injetável

um ou alguns medicamentos considerados potencialmente perigosos pelas suas características de risco, por existir um elevado registo de erros associados ao medicamento ou por esse medicamento conduzir a erros com elevado risco de causar danos graves aos doentes. ${ }^{1,5,8,9}$

Após análise da literatura verificou-se que a utilização de metotrexato oral está associada a um elevado risco de erros de dosagem. Vários países da União Europeia têm desenvolvido medidas para reduzir o risco associado a este medicamento, como exemplo, o uso de lembretes visuais colocados nas caixas de medicação. Contudo, um recente relatório de atualização de segurança da Agência Europeia do Medicamento (EMA), constatou que eventos adversos graves relacionados com overdose, incluindo mortes ainda ocorrem associadas ao uso do metotrexato oral. ${ }^{11,12}$

\section{CONCLUSÃO}

As unidades hospitalares devem desenvolver estratégias e implementar medidas para que o risco de erros associados aos medicamentos de alerta máximo diminua. Uma das estratégias passa pela intervenção de todos os profissionais de saúde conhecerem todos os riscos associados ao uso dos medicamentos de alerta máximo e, também pela formação dos doentes e pela sua educação sobre as formas de administração destes medicamentos, como exemplos, o manuseamento de dispositivos para a monitorização da glicémia, o uso de canetas de insulina, e o manuseamento dos dispositivos para inalação de medicamentos.

Desta forma, todos os envolvidos no processo, desde as direções clínicas, médicos, enfermeiros, farmacêuticos, técnicos de farmácia e assistentes operacionais partiIham a responsabilidade do desenvolvimento e implementação de práticas seguras minimizando a ocorrência de erros com os medicamentos de alerta máximo.
CONFLITOS DE INTERESSE: Os autores declaram não ter qualquer conflito de interesse na realização do presente trabalho.

FONTES DE FINANCIAMENTO: Não houve qualquer fonte de financiamento na realização do presente trabalho.

CONFLICTS OF INTEREST: The authors declare that they have no conflicts of interest.

FINANCIAL SUPPORT: This work has not received any contribution, grant or scholarship.

\section{REFERÊNCIAS}

1. Direcção-Geral da Saúde. Norma I da DGS n014/2015: Medicamentos de Alerta Máximo. Lisboa: DGS; 2015.

2. Instituto para el Uso Seguro de los Medicamentos. Recomendaciones para prevenir los errores causados por confusión en los nombres de los medicamentos. Bol ISMP Brasil. 2012;35: 1-3.

3. Ministerio de sanidad, servicios sociales e igualdad. Proyecto MARC elaboración de una lista de medicamentos de alto riesgo para los pacientes crónicos. Informe 2014 [accessed 5 Apr 2018]; 1: 1-4. Available from: https://www.seguridaddelpaciente.es/resources/documentos/2014/Proyecto MARC_2014.pdf.

4. Cohen Mr, Smetzer JL, Tuohy NR, Kilo Cm. High-alert medications: safeguarding against errors. In: Cohen MR, editor. Medication Errors. 2nd ed. Washington: American Pharmaceutical Association; 2006. p. 317-411.

5. Instituto para Práticas Seguras no Uso de Medicamentos. Medicamentos potencialmente perigosos de uso hospitalar e ambulatorial: Listas atualizadas 2015. Bol ISMP Brasil. 2015;4:110. [accessed 10 May 2018] Available from: http://www.ismp-brasil.org/site/wpcontent/uploads/2015/12/N4N3.pdf.

6. Direcção-Geral da Saúde. Norma I da DGS n020/2014: Medicamentos com nome ortográfico, fonético ou aspeto semeIhantes. Lisboa: DGS; 2014.

7. Lambert BL, Swu-Jane L, Ken-Yu C, Gandhi SK. Similarity as a risk factor in drug name confusion errors: the look-alike (orthographic) and sound-alike (phonological) model. Med Care. 1999;37:1214-25.

8. Mendes AP. Medicamentos potencialmente perigosos em meio hospitalar. Bol Centro Informação Medicamento. 2013;108:1-4.

9. Institute for Safe Medication Practices. ISMP's list of high-alert medications in long-term care (LTC) settings. Huntington Valley (PA):ISMP; 2016. [accessed 1 Mat 2018] Available from: https://www.ismp.org/tools/LTC-High-Alert-List.pdf. Acedido a 1-5- 2018.

10. Winterstein AG, Hatton RC, Gonzalez-Rothi R, Johns TE, Segal R. Identifying clinically significant preventable adverse drug events through a hospital's database of adverse drug reaction reports. Am J Health Syst Pharm. 2002;59:1742-9.

11. European Medicine Agency. EMA reviewing risk of dosing errors with methotrexate. Review prompted by continued reports of overdose. 13 April 2018 EMA/215649/2018. [accessed 22 May 2018]. Available from: http://www.ema.europa. $\mathrm{eu} /$.

12. Instituto para Práticas Seguras no Uso de Medicamentos. Metotrexato de uso oral. Bol ISMP Brasil. 2012;1:2-5. 